\title{
Sometimes they come back: New and old spinal muscular atrophy adults in the era of nusinersen
}

\author{
V. A. Sansone ${ }^{a, *}$, G. Coratti $i^{b, c}, *, M$. C. Pera ${ }^{b, c}$, M. Pane ${ }^{b, c}$, S. Messina ${ }^{d, e}, F$. Salmin ${ }^{a}$, E. Albamonte ${ }^{a}$,

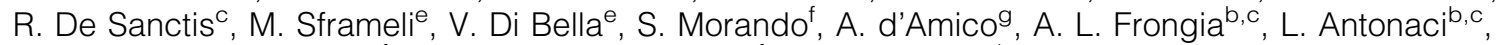 \\ A. Pirola ${ }^{a}$ (D), M. Pedemonte ${ }^{f}$ E. Bertini ${ }^{9}$ (D), C. Bruno and E. Mercurib ${ }^{b, c}$ (ID), on behalf of the Italian ISMAC \\ group $^{, \dagger}$
}

${ }^{\mathrm{a}}$ The NEMO Center in Milan, Neurorehabilitation Unit, ASST Niguarda Hospital, University of Milan, Milan; ${ }^{\mathrm{b}}$ Pediatric Neurology, Università Cattolica del Sacro Cuore, Rome; ${ }^{\mathrm{c} C e n t r o}$ Clinico Nemo, Fondazione Policlinico Universitario Agostino Gemelli IRCCS, Rome; ${ }^{\mathrm{d} D e p a r t m e n t ~ o f ~ C l i n i c a l ~ a n d ~ E x p e r i m e n t a l ~ M e d i c i n e, ~ U n i v e r s i t y ~ o f ~ M e s s i n a, ~ M e s s i n a ; ~}{ }^{\mathrm{e}}$ Nemo SUD Clinical Center, University Hospital 'G. Martino', Messina; ${ }^{\mathrm{f}}$ Center of Translational and Experimental Myology, IRCCS Istituto Giannina Gaslini, Genoa; and ${ }^{\mathrm{g}}$ Department of Neurosciences, Unit of Neuromuscular and Neurodegenerative Disorders, Bambino Gesù Children's Hospital, IRCCS, Rome, Italy

\section{Keywords:}

adult, spinal muscular atrophy, neuromuscular disorders, nusinersen

Received 3 September 2020 Accepted 24 September 2020

European Journal of

Neurology 2021, 28: 602-608

doi: $10.1111 /$ ene. 14567
Background and purpose: Following the commercial availability of nusinersen, there have been a number of new referrals of adults with spinal muscular atrophy (SMA) not regularly followed in tertiary-care centers or enrolled in any disease registry.

Methods: We compared demographics and disease characteristics, including assessment of motor and respiratory function, in regularly followed patients and newcomers subdivided according to the SMA type.

Results: The cohort included 166 adult patients (mean age: 37.09 years): one type I, 65 type II, 99 type III, and one type IV. Of these 166, there were 67 newcomers. There was no significant difference between newcomers and regularly followed patients in relation to age and disease duration. The Hammersmith Functional Motor Scale Expanded and Revised Upper Limb Module scores were higher in the regularly followed patients compared to newcomers in the whole cohort and in both SMA II and II. A difference was also found on ventilatory status $(p=0.013)$ and Cobb's angle $>50^{\circ}(p=0.039)$ between the two subgroups. No difference was found in scoliosis surgery prevalence ( $p$ $>0.05)$.

Conclusions: Our results showed differences between the two subgroups, even if less marked in the type III patients. In the type II patients, there was a higher proportion of newcomers who were in the severe end of the spectrum. Of the newcomers, only approximately a third initiated treatment, as opposed to the $51 \%$ in the regularly followed patients. The identification of patients who were not part of the registries will help to redefine the overall prevalence of SMA and the occurrence of different phenotypes.
Correspondence: E. Mercuri, Largo Agostino Gemelli 8, Rome 00152, Italy (tel.: +39 0630155340; fax: +39 0630153426; e-mail: eugeniomaria.mercuri@unicatt.it).

*V. A. Sansone and G. Coratti are co-first authors.

${ }^{\dagger}$ Group members are listed in the Appendix.

\section{Introduction}

Spinal muscular atrophy (SMA) is an autosomal recessive disease caused by the loss of the SMN1 gene with deficiency of the survival motor neuron protein resulting in progressive motor neuron degeneration, leading to muscle atrophy and weakness [1,2]. The 
advent of clinical trials using different approaches and the subsequent approval of two products, nusinersen and more recently onasemnogene abeparvovec, have completely changed the natural history of the disease. The pivotal studies were mainly performed in infants and young children. The drugs have been approved worldwide for all SMA patients, irrespective of age, probably taking into account that the effect seen in type I infants or in young children with late onset $[3,4]$ may also be observed in older patients, especially in those with a higher SMN2 copy number. Because of this, in some countries nusinersen has been made available for all types of SMA and for all ages, including adults who had not been included in the pivotal studies $[3,4]$. Recent papers on the real-world use of nusinersen in adult patients report a wide range of functional levels at baseline, highlighting the challenges to define treatment effect in such a heterogeneous group [5-7].

The challenges are mainly related to the paucity of natural history data to be used for comparison with treated adult SMA [3-11], but also to an unexpected increase in the number and type of adult SMA patients referred to tertiary-care centers [8-16].

In 2017, as a result of the news reporting the availability of nusinersen as a new treatment on national media and through advocacy groups and social networks [17], many adult SMA patients, unknown to national registries or to the network of tertiary-care centers, became suddenly "visible" as they contacted expert centers to have information on the new treatments in general.

We describe the clinical and demographic features of a large adult cohort with SMA report our experience of the new patients who came to clinic at the time new treatments became available, and try to establish possible differences with the adult patients regularly followed in our clinics.

\section{Methods}

The study is part of a large multicentric study on natural history in spinal muscular atrophy involving five Italian tertiary-care centers. Since June 2018, data have been collected as part of an International Spinal Muscular Atrophy Registry and Consortium [18].

The study was approved by the ethics committee at each center. Written informed consent was obtained for all the patients who agreed to participate.

Demographics and disease characteristics were collected on the last visit. For patients who started nusinersen, the data were collected on the last visit before treatment started. Information on the accessibility to nusinersen treatment was retrieved from the last follow-up visit available.

Adult newcomers were defined as those patients having their first visit at one of the centers, after 2017, with the main purpose of having access or information about the nusinersen treatment.

All patients were assessed using functional measures, such as the Hammersmith Functional Motor Scale Expanded (HFMSE) for SMA and Revised Upper Limb Module (RULM), and respiratory function using forced vital capacity (FVC) in liters and percent predicted [9,19-24]. As type II and III patients are known to be at risk of developing respiratory and nutritional problems $[25,26]$, and to have severe scoliosis, we also systematically collected details of ventilatory and nutritional status and scoliosis surgery.

Newcomers also underwent a nonstructured interview to establish if they were currently or had previously been followed in other tertiary-care centers.

\section{Statistical analysis}

The cohort was subdivided in newcomers and regular follow-up patients and subgrouped by type and motor functional status. Descriptive statistics ( $n$, mean, standard deviation [SD], range) were used between the two populations to summarize data on age, disease duration, ventilatory status, nutritional status, and scoliosis surgery.

The Mann-Whitney $U$ test was used to compare the distribution of patients, mean age, mean disease duration, FVC values, and HFMSE and RULM scores between the two populations and their type and motor functional status. A $\chi^{2}$ test was used to compare ventilatory status, nutritional status, and scoliosis incidence and surgery between the two populations and their type and motor functional status. A $p$ value was set at $<0.05$ for all the analysis.

\section{Results}

\section{Patient population}

At the time of the analysis (March 2020), our datasets included over 500 patients. The adult population included 180 patients ranging in age from 18.02 to 74.39 years (mean: 36.25 , SD: 14.35). Fourteen of the 180 adult patients were enrolled in clinical trials and were not included in the analysis.

The adult cohort for whom data were available includes 166 patients (78 females and 88 males). One was type I, 65/166 were type II, 99/166 were type III, and one was type IV. 


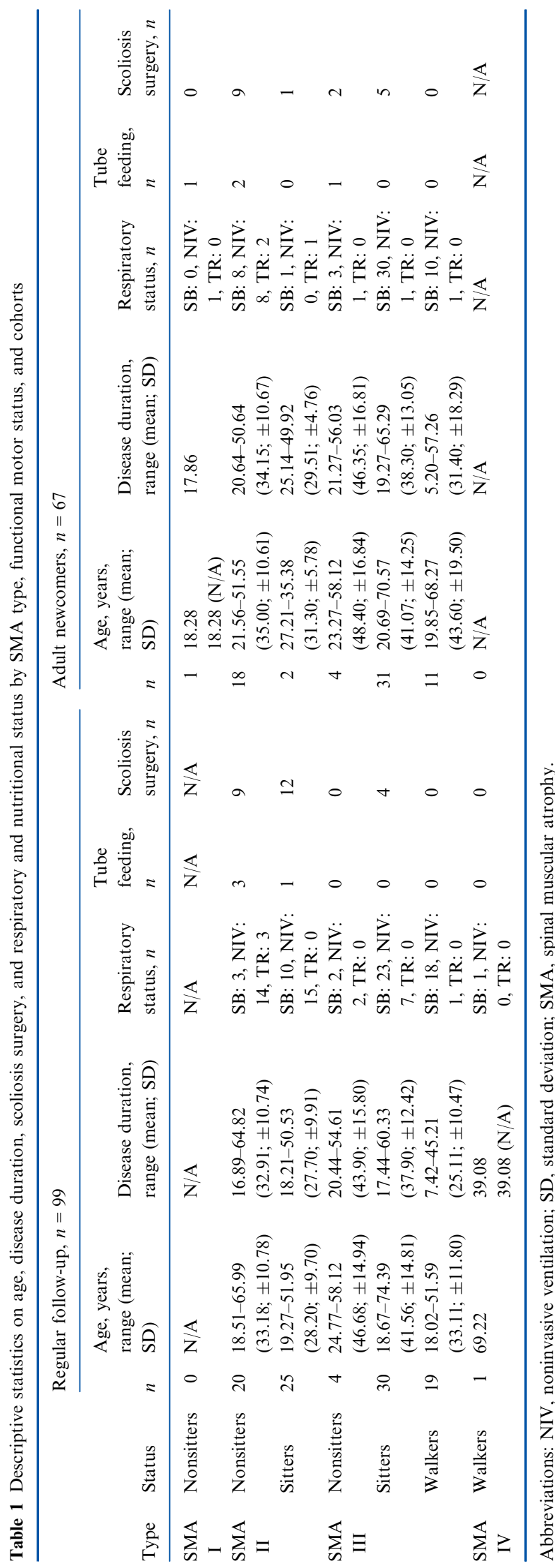

The mean age of the whole cohort was 37.09 years (range: 18.02-74.39 years; SD: 14.19). The SMA I patient was 18.28 year old at the moment of enrollment, the mean age for SMA II was 31.71 years (range: 18.51-65.99 years; SD: \pm 10.43 ), and for SMA III was 40.50 years (range: $18.02-74.39$ years; SD: $\pm 14.94)$. The SMA IV patient was 69.22 years old.

The mean disease duration was 33.56 years (range: 5.20-65.29 years; SD: 12.97) for the whole group, 31.20 years (range: $16.89-64.82$ years; SD: \pm 10.47 ) for SMA II, and 35.31 years (range: 5.20-65.29 years; SD: 14.30) for SMA III.

Of 166 patients, 99 were already regularly followed in our centers. The remaining 67 (34 females and 33 males) came to our clinics with the main purpose of having information on the nusinersen treatment accessibility. None was currently regularly followed in tertiary centers, but all had been followed in pediatric centers in the past. Details of the interval since the last appointment at a tertiary-care center were often not available.

Of the 67 patients, one was type I, 20/67 were type II, and 46/67 were type III.

SMN2 copy number was available in 76 of the 166; 12 had two (seven SMA II, five SMA III), 43 had three (22 SMA II, 21 SMA III), and 21 had four (all SMA III). In nearly half of the (49/99, $49.5 \%)$ regularly followed and in 43/67 (64.2\%) newcomers, SMN2 copy number was not yet available. Of the 43 newcomers, only five $S M N 2$ copy numbers were available at the time of their first access to our centers.

Table 1 reports descriptive statistics on age, mean disease duration, scoliosis surgery, respiratory and nutritional details, subdivided by SMA type and functional status, in the patients previously followed and in the newcomers.

No difference was found on mean age at visit $(p>0.05)$ or mean disease duration $(p>0.05)$ between newcomers and the regularly followed patients.

\section{SMA type and functional status}

In the type II subgroup, there were more patients who had lost the ability to sit among the newcomers $(90 \%)$ compared to the regularly followed patients $(44.4 \%)$ $(p=0.001)$. No difference was found between the type SMA III motor functional subgroups $(p>0.05)$.

The HFMSE and RULM scores were higher in the regularly followed patients compared to newcomers in the whole cohort and in both SMA II and III. The difference reached significance between SMA II newcomers and regularly followed subgroups $(p=0.002$ 


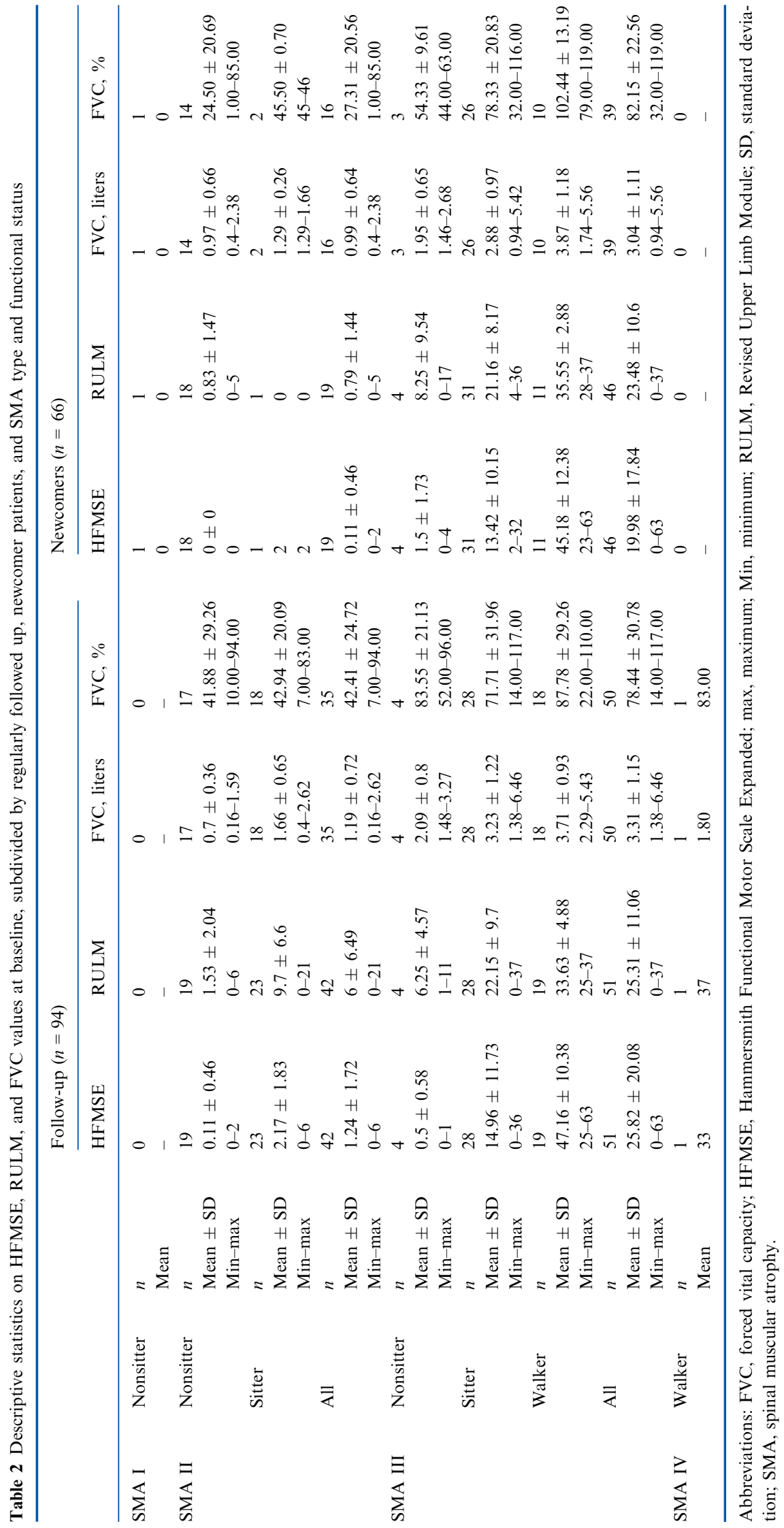


for HFMSE and $p=0.001$ for RULM scores) but not for SMA III patients $(p>0.05)$ (Table 2).

\section{Respiratory function and ventilatory status}

The FVC values in liters and percent predicted were higher in the regularly followed patients compared to newcomers in the whole cohort and in both SMA II and III (Table 2), but the difference did not reach significance.

A difference was found on ventilatory status ( $p=0.013)$ between the two subgroups, with more regularly followed patients using noninvasive ventilation (NIV). Subdividing the cohort by SMA type and functional status, there was also a difference in ventilatory status within the nonsitter SMA II $(p=0.04)$ and sitter SMA III $(p=0.020)$ groups, as in both there were more regularly followed patients using NIV.

Twelve newcomers had awareness of previous abnormal sleep study and/or signs of nocturnal hypoventilation but had previously refused ventilation. Seven of them started ventilation after agreeing to being enrolled in a new follow-up program.

\section{Scoliosis}

The number of patients with a Cobb's angle more than $50^{\circ}$ was lower in the regularly followed patients compared to the newcomers $(p=0.039)$. No difference in scoliosis surgery was found between the two subgroups $(p \geq 0.05)$, as in both groups there was a high number of patients who had refused scoliosis surgery.

\section{Nutritional status}

No difference in nutritional status was found between the two populations $(p \geq 0.05)$.

\section{Nusinersen treatment}

Seventy-two of the 166 patients were on nusinersen (43.37\%): 21 of the $67(31.34 \%)$ newcomers and 51 of the $99(51.51 \%)$ regularly followed patients.

The mean treatment duration at the time of the analysis was 528.57 days $( \pm 166.83$; 64-679) for the 21 newcomers and 548.09 days $( \pm 144.40 ; 202-799)$ for the 51 regularly followed patients.

\section{Discussion}

In this study we present a snapshot of a large cohort of Italian adult patients with SMA, focusing on those who were, until recently, not followed in a tertiary- care center. These patients were also not part of family's associations or other advocacy groups, and, despite having a diagnosis of SMA, until recently had not been detected by any registry or natural history study.

In our current dataset, 67 of the 166 adult patients were newcomers who approached our tertiary- care centers because they were interested in possible treatment with nusinersen. The new referrals included mainly adult patients who were managed at home with local support, such as some severe type II patients and even a type I patient who had survived with permanent ventilation beyond the age of 18 years.

With few exceptions, all had received their diagnosis when they were children and had previously been followed in tertiary-care centers. However, when transitioning from the pediatric to the adult age, most of them had gradually lost interest in attending specialist centers. Patients often complained that the assessments performed by adult neurologists were less detailed and there was less physiotherapist input compared to the pediatric setting.

It is of interest that although the new patients did not differ significantly from those regularly followed in relation to age, disease duration, and other parameters, some differences between the two groups could be noted. Both groups had a widespread range of age and functional abilities, confirming the wide heterogeneity that can be found in adults affected by SMA. In the type II group, there was a higher proportion of newcomers who were in the severe end of the spectrum of type II (18/20: $90.00 \%)$, compared to the regularly followed patients $(20 / 45 ; 44.44 \%)$. These were patients who had lost the ability to sit independently, had very residual motor function, and had an overall severe motor phenotype compared to the type II patients who had retained the ability to sit. Not surprisingly, within type II, the difference in functional status is also reflected by a significant difference in HFMSE and RULM scores.

Within the type II group, all patients had developed severe scoliosis since childhood, and only few had opted for scoliosis surgery when this was suggested, with no difference between newcomers and regularly followed patients. In both groups, patients reported that at the time surgery was discussed, they felt that the risk of a general anesthesia and a long procedure, together with the possible loss of some functional abilities, contributed to their refusal.

A significant difference was in contrast found on respiratory management. There was a limited number of patients using any form of ventilation in the newcomers group, despite many of them being aware of 
clinical signs and, in some cases, also of abnormal sleep studies. Patients reported that they felt that ventilation was not needed as they felt in good health, and they did not want to wear a mask at night. In seven patients we were able to start ventilation once they decided to be followed regularly.

The differences between regularly followed patients and newcomers were less marked in the type III patients. The two subgroups had similar percentages of patients who were still able to walk $(35.85 \%$ vs. $23.91 \%)$, had become sitters $(56.60 \%$ vs. $67.39 \%)$, or had even lost the ability to sit ( $7.55 \%$ vs. $8.69 \%)$.

When we analyzed functional scores and FVC in type III patients, the regularly followed patients had consistently better values, but the difference was not significant. Interestingly, as also observed in type II, four of the eight nonsitter type III patients had previously opted for not using ventilation that was initiated after they agreed to remain in a follow-up program. In general, regularly followed patients had a much better adherence to respiratory care recommendations and posturing compared to the newcomers. In contrast, there was little difference for aspects that were considered and discussed at the time the patients were in the pediatric age, such as the possibility of scoliosis surgery. Unfortunately, many adult patients refused to take a new x-ray, and we do not have an accurate estimate of the severity of scoliosis; however, from physical examination, the level of scoliosis was obviously different, with many newcomers having an inappropriate wheelchair posturing that had not been revised for several years.

It is of interest that although most of these patients received new referrals with the main aim to discuss the possibility to perform therapy with nusinersen, only approximately a third initiated treatment, as opposed to the $51 \%$ of the regularly followed patients. In 24 cases, this was due to surgery $(n=17)$ or to a very severe scoliosis $\left(>70^{\circ}\right)(n=7)$ that would have required the use of imaging with radiation. In other cases, patients chose to wait and see, as there was little experience with nusinersen in adults. Interestingly, with few exceptions, patients agreed to be followed regularly even if they did not start treatment.

The study also prompted us to develop patient-reported outcome measures and other tools to better understand adult patients' attitudes toward care. These measures were not available at the time the patients entered our registry, but all of the newcomers were asked the reasons for having discontinued care at tertiary-care centers. Type II patients who had lost the ability to sit and type III patients who lost ambulation often reported that after losing several motor abilities by the time of puberty, they felt demotivated to continue follow-up, as despite all of their efforts they were still steadily deteriorating. Traveling and additional resources contributed to outweigh the benefits of visiting their referring neurologist. After leaving the tertiary-care center, most of them chose to be followed by their local physicians, often from the rehabilitation centers for specific needs such as a change in a wheelchair or other orthosis, had seasonal general check-ups or other SMA-unrelated medical needs, and only a few saw a pneumologist or had received a multidisciplinary approach.

Another important issue is that despite the extensive literature on $S M N 2$ copy number, many adult patients who had the diagnosis of SMN1 mutations many years ago, when the copy number was not automatically determined, do not have this information available. All patients were asked to consent for the test, but surprisingly, a high number declined, and others decided to postpone it for the time when other blood tests were needed. The number of patients with SMN2 copy number is now rapidly increasing, and this will help to establish whether the number of $S M N 2$ copies correlates to the degree of motor disability and progression in this older population.

Our results confirm previous studies reporting a large phenotypic variability in adults affected by SMA and suggest that further efforts should be made to identify and follow all SMA patients including those who are not part of the registries or followed in tertiary-care centers. The identification of these cases will lead to redefining the overall prevalence of SMA and the occurrence of different phenotypes. A better awareness of the progression of the disease according to age and functional level will also help to set up realistic expectations in relation to new treatments or other types of intervention.

\section{Acknowledgements}

The study received funding from the Italian Telethon (GSP13002). From June 2018, data were available from the International SMA Registry, partly funded with a contribution from Biogen.

\section{Disclosure of conflicts of interest}

Dr. Sansone, Dr. Coratti, Dr. De Sanctis, Dr. Pane, Dr. Messina, Dr. D'Amico, Dr. Albamonte, Dr. Bertini, Dr. Bruno, and Dr. Mercuri report personal fees from Biogen S.R.L. outside the submitted work. Dr. Pera, Dr. Coratti, Dr. Sframeli, Dr. De Sanctis, Dr. Bertini, Dr. Messina, Dr. Mercuri reports personal fees from Roche S.R.L. outside the submitted 
work. Dr. Sansone, Dr. De Sanctis, Dr. Coratti, Dr. Pane, Dr. Messina, Dr. Bertini, and Dr. Mercuri report personal fees from AveXis S.R.L. outside the submitted work.

\section{Data availability statement}

The data that support the findings of this study are available from the corresponding author upon reasonable request.

\section{References}

1. Lefebvre S, Burglen L, Reboullet S, et al. Identification and characterization of a spinal muscular atrophy-determining gene. Cell 1995; 80: 155-165.

2. D’Amico A, Mercuri E, Tiziano FD, Bertini E. Spinal muscular atrophy. Orphanet J Rare Dis 2011; 6: 71.

3. Mercuri E, Darras BT, Chiriboga CA, et al. Nusinersen versus sham control in later-onset spinal muscular atrophy. $N$ Engl J Med 2018; 378: 625-635.

4. Finkel RS, Mercuri E, Darras BT, et al. Nusinersen versus sham control in infantile-onset spinal muscular atrophy. $N$ Engl J Med 2017; 377: 1723-1732.

5. Stolte B, Totzeck A, Kizina $\mathrm{K}$, et al. Feasibility and safety of intrathecal treatment with nusinersen in adult patients with spinal muscular atrophy. Ther Adv Neurol Disord. 2018; 11: 1756286418803246.

6. Hagenacker $\mathrm{T}$, Wurster CD, Gunther R, et al. Nusinersen in adults with $5 \mathrm{q}$ spinal muscular atrophy: a noninterventional, multicentre, observational cohort study. Lancet Neurol 2020; 19: 317-325.

7. Mercuri E, Sansone V. Nusinersen in adults with spinal muscular atrophy: new challenges. Lancet Neurol 2020; 19: $283-4$.

8. Wijngaarde CA, Stam M, Otto LAM, et al. Populationbased analysis of survival in spinal muscular atrophy. Neurology 2020; 94: e1634-e44.

9. Wijngaarde CA, Veldhoen ES, van Eijk RPA, et al. Natural history of lung function in spinal muscular atrophy. Orphanet J Rare Dis 2020; 15: 88.

10. Elsheikh B, King W, Peng J, et al. Outcome measures in a cohort of ambulatory adults with spinal muscular atrophy. Muscle Nerve 2020; 61: 187-191.

11. Elsheikh B, Prior T, Zhang X, et al. An analysis of disease severity based on SMN2 copy number in adults with spinal muscular atrophy. Muscle Nerve 2009; 40: 652-656.

12. Bertini E, Burghes A, Bushby K, et al. 134th ENMC International Workshop: outcome measures and treatment of spinal muscular atrophy, 11-13 February 2005, Naarden, The Netherlands. Neuromuscul Disord. 2005; 15: $802-816$.

13. Wadman RI, Wijngaarde CA, Stam M, et al. Muscle strength and motor function throughout life in a crosssectional cohort of 180 patients with spinal muscular atrophy types 1c-4. Eur J Neurol 2018; 25: 512-518.

14. Finkel R, Bertini E, Muntoni F, Mercuri E, Group ESWS. 209th ENMC International Workshop: outcome measures and clinical trial readiness in spinal muscular atrophy 7-9 November 2014, Heemskerk, The Netherlands. Neuromuscul Disord 2015; 25: 593-602.

15. Montes J, McDermott MP, Mirek E, et al. Ambulatory function in spinal muscular atrophy: age-related patterns of progression. PLoS One 2018; 13: e0199657.

16. Mercuri E, Finkel R, Montes J, et al. Patterns of disease progression in type 2 and 3 SMA: implications for clinical trials. Neuromuscul Disord 2016; 26: 126-131.

17. Pacione M, Siskind CE, Day JW, Tabor HK. Perspectives on spinraza (nusinersen) treatment study: views of individuals and parents of children diagnosed with spinal muscular atrophy. J Neuromuscul Dis 2018; 6: $119-131$.

18. Mercuri E, Finkel R, Scoto M, et al. Development of an academic disease registry for spinal muscular atrophy. Neuromuscul Disord 2019; 29: 794-799.

19. Main M, Kairon H, Mercuri E, Muntoni F. The Hammersmith functional motor scale for children with spinal muscular atrophy: a scale to test ability and monitor progress in children with limited ambulation. Eur $J$ Paediatr Neurol 2003; 7: 155-159.

20. Mazzone E, Montes J, Main M, et al. Old measures and new scores in spinal muscular atrophy patients. Muscle Nerve 2015; 52: 435-437.

21. Mercuri E, Messina S, Battini R, et al. Reliability of the Hammersmith functional motor scale for spinal muscular atrophy in a multicentric study. Neuromuscul Disord 2006; 16: 93-98.

22. Mazzone ES, Mayhew A, Montes J, et al. Revised upper limb module for spinal muscular atrophy: development of a new module. Muscle Nerve 2016;55: 869-874.

23. Pera MC, Coratti G, Mazzone ES, et al. Revised upper limb module for spinal muscular atrophy: 12 month changes. Muscle Nerve 2019; 59: 426-430.

24. Glanzman AM, O'Hagen JM, McDermott MP, et al. Validation of the expanded hammersmith functional motor scale in spinal muscular atrophy type II and III. $J$ Child Neurol 2011; 26: 1499-1507.

25. Finkel RS, Mercuri E, Meyer OH, et al. Diagnosis and management of spinal muscular atrophy: part 2: pulmonary and acute care; medications, supplements and immunizations; other organ systems; and ethics. Neuromuscul Disord 2018; 28: 197-207.

26. Mercuri E, Finkel RS, Muntoni F, et al. Diagnosis and management of spinal muscular atrophy: part 1: recommendations for diagnosis, rehabilitation, orthopedic and nutritional care. Neuromuscul Disord 2018; 28: 103-115.

\section{Appendix. Italian ISMAC group}

Alessandra Di Bari, Amelia Signorino, Antonella Longo, Paola Tacchetti, Noemi Brolatti, Diletta Rossi, Chiara Bravetti, Simona Lucibello, Lavinia Fanelli, Nicola Forcina, Giulia Norcia, Sara Carnicella, Katia Agata Patanella, Daniela Leone, Concetta Palermo, Beatrice Berti, Felice Catania, Andrea Colombo, Aurora Bozzardi, Gloria Ferrantini, Gianluca Vita. 